\title{
Some Results on Degree of Vertices in Semitotal-Block Graph and Total-Block Graph
}

\author{
Bhavanari Satyanarayana ${ }^{1}$, \\ Devanaboina Srinivasulu ${ }^{2}$ \\ ${ }_{1,2}$ Department of \\ MathematicsAcharyaNagarjuna \\ University \\ Nagarjuna Nagar-522 510 \\ Andhra Pradesh, India
}

\author{
Kuncham Syam Prasad \\ Department of Mathematics, MIT, \\ Manipal University, \\ Manipal-576 104, India
}

\author{
Eswaraiah Setty S. \\ SGS College, \\ Jaggaiahpet, Krishna Dist. \\ Andhra Pradesh, India
}

\begin{abstract}
We considersemitotal-block graph, total-block graph of a graph $G$ (respectively, denoted as $T_{b}(G), T_{B}(G)$ ). We prove that the number of edges in a semitotal-block graph of a given graph $\mathrm{G}$ is equal to $|\mathrm{E}(\mathrm{G})|+\left|\mathrm{V}\left(\mathrm{B}_{1}\right)\right|+\left|\mathrm{V}\left(\mathrm{B}_{2}\right)\right|+\ldots+\left|\mathrm{V}\left(\mathrm{B}_{\mathrm{m}}\right)\right|$, where $B_{1}, B_{2}, \ldots, B_{m}$ are the blocks of $G$. Further, we obtain that $T_{B}(G)$ is the ring sum of $T_{b}(G)$ and the block graph $\mathrm{B}(\mathrm{G})$. We introduce the concept "vertex-block graph (denoted by $B_{v}(G)$ of $G$ )", andwe prove that $T_{b}(G)$ is the ring sum of $G$ and $B_{v}(G)$. We also present some related fundamental results along with illustrations.
\end{abstract}

\section{General Terms}

Graph Theory, Characterization of different Block Graphs.

\section{Keywords}

Degree of vertex, Semitotal-block graph, Total-block graph.

\section{INTRODUCTION}

A finitegraph $\mathrm{G}=(\mathrm{V}, \mathrm{E})$ consists of a finite nonempty set of objects, $\mathrm{V}=\left\{\mathrm{v}_{1}, \mathrm{v}_{2}, \ldots\right\}$ called vertices and another finite set, $\mathrm{E}=\left\{\mathrm{e}_{1}, \mathrm{e}_{2}, \ldots\right\}$ of elements called edges such that each edge $e_{k}$ is identified with an unordered pair $\left\{\mathrm{v}_{\mathrm{i}}, \mathrm{v}_{\mathrm{j}}\right\}$ of vertices. An edge associated with a vertex pair $\left\{\mathrm{v}_{\mathrm{i}}, \mathrm{v}_{\mathrm{i}}\right\}$ is called a selfloop. The number of edges associated with the vertex is the degree of the vertex, and $\delta(\mathrm{v})$ denotes the degree of the vertex v. If there is more than one edge associated with a given pair of vertices, then these edges are called parallel edges (or) multiple edges. A graph that has does not have self-loop or parallel edges called a simple graph. Two vertices are said to be adjacent if they are the end vertices of the same edge. A finite alternating sequence of vertices and edges (no repetition of edge allowed) beginning and ending with vertices such that each edge is incident with the vertices preceding and following it, is called a walk and an open walk in which no vertex appears more than once, is called a path. A graph said to be connected if there is at least one path between every pair of vertices in $\mathrm{G}$, otherwise it is called disconnected. In a connected graph, a vertex whose removal disconnects the graph is called a cut-vertex. The authors in [9] studied the cut vertices and a special type of symmetry in graphs. The $n$-cube defined as, for a set $\mathrm{X}$ with $|\mathrm{X}|=\mathrm{n}$ and $\wp(\mathrm{X})$ be its power set. Then a graph having $\wp(X)$ as its vertex set; and there is an edge between two vertices $\mathrm{A}, \mathrm{B}$ if and only if $|\mathrm{A} \Delta \mathrm{B}|=1$ where $\mathrm{A} \Delta \mathrm{B}=(\mathrm{A} \backslash \mathrm{B}) \cup(\mathrm{B} \backslash \mathrm{A})$, is called the $n$-cube. The graph $n$-cube is characterized and obtained an isomorphism theorem in $[6,7$, and 10$]$.

For any two graphs $\mathrm{G}_{1}=\left(\mathrm{V}_{1}, \mathrm{E}_{1}\right)$ and $\mathrm{G}_{2}=\left(\mathrm{V}_{2}, \mathrm{E}_{2}\right)$, we define their union as the graph $\mathrm{G}=(\mathrm{V}, \mathrm{E})$ where $\mathrm{V}=$ $\mathrm{V}_{1} \cup \mathrm{V}_{2}$ and $\mathrm{E}=\mathrm{E}_{1} \cup \mathrm{E}_{2}$, their intersectionwhen $\mathrm{V}_{1} \cap$ $\mathrm{V}_{2} \neq \phi$ is defined as the graph $\mathrm{G}=(\mathrm{V}, \mathrm{E})$ where $\mathrm{V}=\mathrm{V}_{1} \cap$ $\mathrm{V}_{2}$ and $\mathrm{E}=\mathrm{E}_{1} \cap \mathrm{E}_{2}$, and the ring sum $\mathrm{G}_{1} \oplus \mathrm{G}_{2}$ of two graphs $\mathrm{G}_{1}$ and $G_{2}$ is defined as the graph $\mathrm{G}=(\mathrm{V}, \mathrm{E})$ where $\mathrm{V}=\mathrm{V}_{1} \cup \mathrm{V}_{2}$ and $\mathrm{E}=\left(\mathrm{E}_{1} \cup \mathrm{E}_{2}\right) \backslash\left(\mathrm{E}_{1} \cap \mathrm{E}_{2}\right)$.

In this paper, we consider only finite simple graphs.

For the remaining fundamental definitions and results which are used in the paper, we refer [1,2, 5, and 7].

\section{SEMITOTAL-BLOCK GRAPHS}

In this section, we define semitotal-block graphs and provide some examples. We prove that the number of edges in a semitotal-block graph is equal to the sum of the edges in a graph and the number of vertices in all the blocks of a graph.

1.1Definition [2]: A connected non-trivial graph having no cut point is a block. A block of a graph is a sub-graph that is a block and is maximal with respect to this property.

1.2 Note: The set of all Blocks of $G$ is denoted by $S B(G)$

1.3 Example: (i). Consider the graph $\mathrm{G}$ in figure 1 .

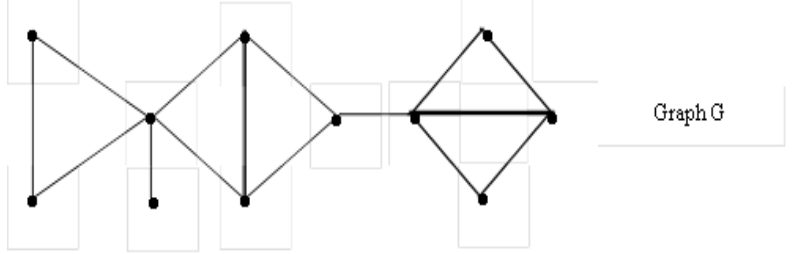

Figure 1

There are five blocks in $G$. They are $B_{1}(G), B_{2}(G), B_{3}(G)$, $B_{4}(G), B_{5}(G)$. These blocks are shown in figure 2 .

In this example, $S B(G)=\left\{B_{1}(G), B_{2}(G), B_{3}(G), B_{4}(G)\right.$, $\left.\mathrm{B}_{5}(\mathrm{G})\right\}$. 


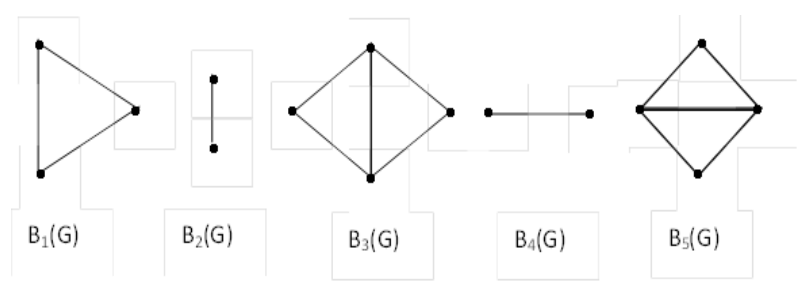

Figure 2

(ii) Observe that n-cube contains no cut point; and so it contains no other blocks except itself. That is, n-cube has just one block.

1.4 Definition [4]: The semitotal-block graph (denoted by $T_{b}(G)$ ) of a given graph $G$ is defined as the graph having point set $V(G) \cup B(G)$, with two points adjacent if they correspond to two adjacent points of $\mathrm{G}$ or one corresponds to a block Bof $\mathrm{G}$ and other to a point $\mathrm{v}$ of $\mathrm{G}$ and $\mathrm{v}$ in $\mathrm{B}$

1.5 Example: Consider the graph $\mathrm{G}$ given in Figure 3.

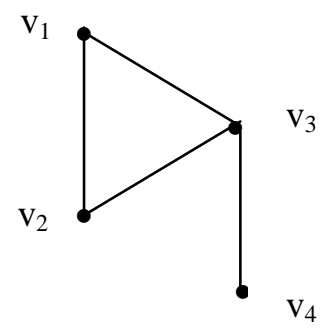

Figure 3

There are two blocks $B_{1}$ and $B_{2}$ of $G$ are given in figure 4 .

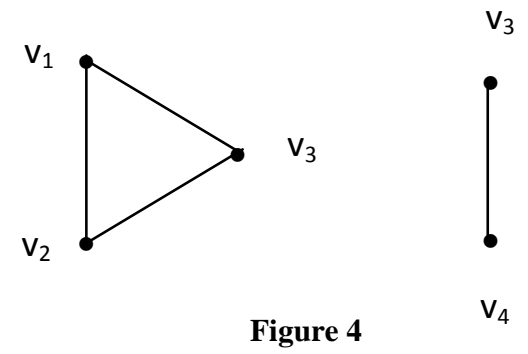

Then $B(G)=\left\{\begin{array}{lll}B_{1} & B_{2}\end{array}\right\}$. We construct the semi total-block graph $T_{b}(G)$ Now $V\left(T_{b}(G)\right)=$ The vertex set of $T_{b}(G)=V(G)$ $\cup B(G)=\left\{v_{1}, v_{2}, v_{3}, v_{4}, B_{1}, B_{2}\right\}$. Following the definition, we draw the semitotal-block graph $T_{b}(G)$ of $G$ is given in figure 5 .

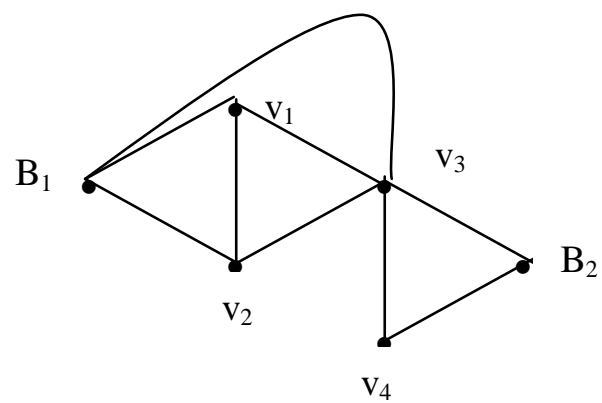

Figure 5
1.6 Note: Let $G$ be a graph. By the definition of $T_{b}(G)$, it follows that every edge in $G$ is also an edge in $T_{b}$. Therefore $\mathrm{E}(\mathrm{G}) \subseteq \mathrm{E}\left(\mathrm{T}_{\mathrm{b}}(\mathrm{G})\right)$. Thus $\mathrm{G}$ is a subgraph of $\mathrm{T}_{\mathrm{b}}(\mathrm{G})$.

1.7 Definition: Let $\mathrm{G}$ be a graph and $\mathrm{B}$ a block in $\mathrm{G}$. An edge e in $T_{b}(G)$ is said to be an edge related to block $\mathrm{B}$ if one of the end points of $e$ is $B$.

\subsection{Lemma: Let B be a block in the given graph G. Then}

(i). The degree of the vertex B (of the semitotal-block graph) is equal to the number of vertices in the block $B$ (of the given graph G).

(ii). The number of edges in $T_{b}(G)$ related to block $B$ is $|V(B)|$.

Proof: (i) Suppose that the block B consists of ' $k$ ' vertices and $\mathrm{V}(\mathrm{B})=\left\{\mathrm{v}_{1}, \mathrm{v}_{2}, \ldots \mathrm{v}_{\mathrm{k}}\right\}$.

Since each $\mathrm{v}_{\mathrm{i}}$ is in the block $\mathrm{B}$, by the definition of semitotalblock graph, we get an edge between the vertex $\mathrm{v}_{\mathrm{i}}$ and $\mathrm{B}$ (in the semitotal-block graph $T_{b}(G)$ ). This is true for all $\mathrm{i}$ with 1 $\leq i \leq k$. Thus there are ' $k$ ' edges with end point $B$ in $T_{b}(G)$. Any edge with an end point $B$ in $T_{b}(G)$ is obtained in this way. Hence we get (i).

(ii) From (i), we get that degree of $B\left(\right.$ in $\left.T_{b}(G)\right)$ is k.

1.9 Theorem: Let G be a connected graph. Then

$\left|E\left(T_{b}(G)\right)\right|=|E(G)|+\sum_{i=1}^{m}\left|V\left(B_{i}\right)\right|$, where $B_{1}, B_{2}, \ldots B_{m}$ are the blocks of $\mathrm{G}$.

Proof: Suppose $\mathrm{SB}(\mathrm{G})=\left\{\mathrm{B}_{1}, \mathrm{~B}_{2}, \ldots, \mathrm{B}_{\mathrm{m}}\right\}$. Let e be an edge in $\mathrm{T}_{\mathrm{b}}(\mathrm{G})$.

If $\mathrm{e}$ is an edge between two vertices in $\mathrm{V}(\mathrm{G})$, then $\mathrm{e} \in \mathrm{E}(\mathrm{G})$. Otherwise, the edge formed in between a vertex and a block $B_{i}$ (of $\left.G\right)$. Let $B$ be a block in $G$ with $k$ vertices.

By Lemma 1.8, the number of edges related to block B is $|\mathrm{V}(\mathrm{B})|=\mathrm{k}$. Therefore the number of distinct edges (related to block $B)$ that exist in $T_{b}(G)$ is $|V(B)|$.

This is true for each block B in G. Thus there are $\sum_{i=1}^{m}\left|V\left(B_{i}\right)\right|$ edges in $T_{b}(G)$ that are related to different blocks of $G$. Hence the number of edges in $T_{b}(G)$ is $|E(G)|+$ $\sum_{\mathrm{i}=1}^{\mathrm{m}}\left|\mathrm{V}\left(\mathrm{B}_{\mathrm{i}}\right)\right|$. The proof is complete.

1.10. Theorem: Let $v$ be a vertex in a given graph $\mathrm{G}$, then the degree of $v$ in $T_{b}(G)=\delta(v)+\mid\{B \mid B$ is a block in $G$ such that $\mathrm{v}$ lies in $\mathrm{B}\} \mid$. 
1.11 Corollary: Let $\mathrm{G}$ be a graph and $\mathrm{v}$ a vertex in $\mathrm{G}$. Then

(i) degree of $v$ in $T_{b}(G) \geq$ degree of $v$ in $G$

(ii) degree of $\mathrm{v}$ in $\mathrm{T}_{\mathrm{b}}(\mathrm{G})=$ degree of $\mathrm{v}$ in $\mathrm{G}$

$\Leftrightarrow \mathrm{v}$ is not contained in any block of $\mathrm{G}$.

Observe that every vertex lies in a block. So the degree of $\mathrm{v}$ in $T_{b}(G) \neq$ degree of $v$ in $G$. Hence degree of $v$ in $T_{b}(G)>$ degree of $\mathrm{v}$ in $\mathrm{G}$.

1.12 Definition: Let $\mathrm{G}$ be a graph. The vertex-block graph (denoted by $B_{v}(G)$ ) of $G$ is defined as follows:

(i) $\mathrm{V}\left(\mathrm{B}_{\mathrm{v}}(\mathrm{G})\right)=\mathrm{V}(\mathrm{G}) \cup \mathrm{SB}(\mathrm{G})$

(ii) $\mathrm{E}\left(\mathrm{B}_{\mathrm{v}}(\mathrm{G})\right)=\{\overline{x y} \mid \mathrm{x} \in \mathrm{V}(\mathrm{G})$ and $\mathrm{y} \in \mathrm{SB}(\mathrm{G})$ such that $\mathrm{x}$ is a vertex of the block $y$.

1.13 Note: (i) $B_{v}(G)$ is a spanning subgraph of $T_{b}(G)$.

(ii) $G$ is not a spanning subgraph of $T_{b}(G)$ if $G$ contains a block.

1.14 Theorem: $T_{b}(G)=G \oplus B_{v}(G)$.

Proof: Let $\mathrm{G}$ be a graph. Since $\mathrm{G}$ and $\mathrm{B}_{\mathrm{v}}(\mathrm{G})$ are subgraphs of $T_{b}(G)$, it follows that $G \cup B_{v}(G) \subseteq T_{b}(G)$.

By the definition of $T_{b}(G)$, every edge in $T_{b}(G)$ is either in $G$ or in $B_{v}(G)$. So $T_{b}(G) \subseteq G \cup B_{v}(G)$. Hence $T_{b}(G)=G$ $\cup B_{v}(G)$. By the definition of $B_{v}(G)$, no edge of $G$ is in $B_{v}(G)$. Therefore Hence $\mathrm{E}\left(\mathrm{G} \oplus \mathrm{B}_{\mathrm{v}}(\mathrm{G})\right)=\left(\mathrm{E}(\mathrm{G}) \backslash \mathrm{E}\left(\mathrm{B}_{\mathrm{v}}(\mathrm{G})\right)\right) \cup$ $\left(E\left(B_{v}(G)\right) \backslash E(G)\right)=E(G) \cup E\left(B_{v}(G)\right)$. Hence $G \oplus B_{v}(G)=G$ $\cup B_{v}(G)$, which shows that $T_{b}(G)=G \oplus B_{v}(G)$.

\section{TOTAL BLOCK GRAPH}

In this section, we study 'total-block graph' and related results with illustrations.

2.1 Definition: The total-block graph (denoted as $\mathrm{T}_{\mathrm{B}}(\mathrm{G})$ ) of a given graph $\mathrm{G}$ is defined as the graph having the point set $V(G) \cup B(G)$, with two points adjacent if they corresponds to either two adjacent points of $G$ or two blocks of $G$ which have a common cut point or one corresponds to a block $B_{i}$ of $G$ and the other to a point $v_{j}$ of $G$ with $v_{j}$ is in $B_{i}$.

2.2 Example: A graph $G$ and its total-block graph $T_{B}(G)$ is given in figure 6 and figure 7 respectively.

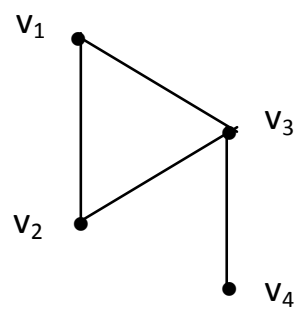

Figure 6

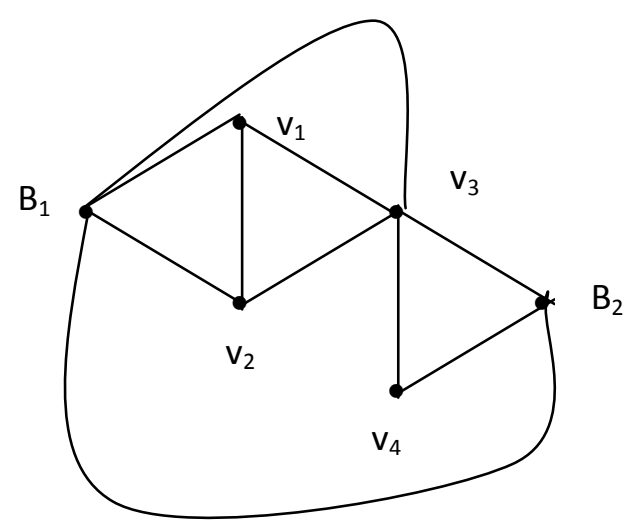

Figure 7

2.3 Note: Let $G$ be a graph. By the definition of $T_{B}(G)$, it follows that every edge of $G$ is in $T_{B}(G)$. So $E(G) \subseteq$ $E\left(T_{B}(G)\right)$. Thus $G$ is a subgraph of $T_{B}(G)$.

2.4Definition: Two blocks $B_{1}$ and $B_{2}$ in a given graph $G$ are said to be adjacentblocks if they have a common cut vertex.

2.5 Theorem: (i) The number of edges in $T_{B}(G)$ related to block $B$ is equal to $|\mathrm{V}(\mathrm{B})|+$ (the number of adjacent blocks to B).

(ii) The degree of $B$ in $T_{B}(G)=|V(B)|+$ (the number of adjacent blocks to B).

Proof: Let B be a block and e be an edge in $T_{B}(G)$ related to block B

Then either $\mathrm{e}=\overline{\mathrm{vB}}$ for some $\mathrm{v} \in \mathrm{B}$ or $\mathrm{e}=\overline{\mathrm{BB}_{1}}$, for some adjacent block $\mathrm{B}_{1}$ of $\mathrm{B}$.

The number of edges of the form $\overline{\mathrm{vB}}$ is $|\mathrm{V}(\mathrm{B})|$. The number of edges of the form $\overline{\mathrm{BB}_{i}}$, is equal to the number of distinct blocks $B_{i}$, which are adjacent to $B$.

Hence the number of edges in $T_{B}(G)$ related to block $B$ is equal to $|\mathrm{V}(\mathrm{B})|+($ the number of adjacent blocks to $\mathrm{B})$. Thus we get (i).

(ii). $\mathrm{F} \quad \mathrm{v}_{2}$ fron

$2.6 \mathrm{~L} . \quad$ in $\left(_{\mathrm{L}} \quad\right.$ : G _.. T. The block graph (denoted by $\mathrm{B}(\mathrm{G})$ ) is defined as follows:

$\mathrm{V}(\mathrm{B}(\mathrm{G}))=\mathrm{SB}(\mathrm{G})$, the set of all blocks of $\mathrm{G}$; and $\mathrm{E}(\mathrm{B}(\mathrm{G}))=$ $\left\{\overline{\mathrm{B}_{1} \mathrm{~B}_{2}} \mid \mathrm{B}_{1}, \mathrm{~B}_{2} \in \mathrm{V}(\mathrm{B}(\mathrm{G}))\right.$ and $\mathrm{B}_{1}$ and $\mathrm{B}_{2}$ have a common cut vertex .

2.7 Remark: Let $\mathrm{G}$ be a graph and $\mathrm{B}$ be a block. Then degree of $B$ in $B(G)$, (the block graph) is equal to the number of distinct adjacent blocks to $\mathrm{B}$ in $\mathrm{G}$.

The following theorem states a relation between the graphs: total-block graph; semitotal-block graph; vertex-block graph; and block graph. 
2.8 Theorem: For a connected graph $\mathrm{G}$, (i) $\mathrm{T}_{\mathrm{B}}(\mathrm{G})=\mathrm{T}_{\mathrm{b}}(\mathrm{G}) \oplus$ $\mathrm{B}(\mathrm{G})$, and

(ii) $T_{B}(G)=G \oplus B_{v}(G) \oplus B(G)$.

Proof: By the definition of $\mathrm{T}_{B}(\mathrm{G})$, it follows that $\mathrm{T}_{b}(\mathrm{G})$ and $B(G)$ are subgraphs of $T_{B}(G)$.

$\mathrm{V}\left(\mathrm{T}_{\mathrm{B}}(\mathrm{G})\right)=\mathrm{V}(\mathrm{G}) \cup \mathrm{SB}(\mathrm{G})=(\mathrm{V}(\mathrm{G}) \cup \mathrm{SB}(\mathrm{G})) \cup \mathrm{SB}(\mathrm{G})$ (by idempotent and associative laws of sets $)=V\left(T_{b}(G)\right) \cup$ $\mathrm{V}(\mathrm{B}(\mathrm{G}))$

Let $\mathrm{s}$ be an edge in $\mathrm{T}_{\mathrm{B}}(\mathrm{G})$. Then $\mathrm{s} \in \mathrm{E}(\mathrm{G})$ or $\mathrm{s}=\overline{\mathrm{vB}}$ for some $\mathrm{v}$ $\in \mathrm{V}(\mathrm{G}), \mathrm{B} \in \mathrm{SB}(\mathrm{G})$ with $\mathrm{v} \in \mathrm{B}$ or $\mathrm{s}=\overline{\mathrm{B}_{1} \mathrm{~B}_{2}}$ for some $\mathrm{B}_{1}$, $B_{2} \in \mathrm{SB}(\mathrm{G})$ with $\mathrm{B}_{1}, \mathrm{~B}_{2}$ are adjacent blocks in $\mathrm{G}$.

Now $\mathrm{s} \in \mathrm{E}(\mathrm{G})$ or $\mathrm{s} \in \mathrm{E}\left(\mathrm{B}_{\mathrm{v}}(\mathrm{G})\right)$ or $\mathrm{s} \in \mathrm{E}(\mathrm{B}(\mathrm{G})) \Rightarrow \mathrm{s} \in \mathrm{E}(\mathrm{G})+$ $\mathrm{E}\left(\mathrm{B}_{\mathrm{v}}(\mathrm{G})\right)=\mathrm{E}\left(\mathrm{T}_{\mathrm{b}}(\mathrm{G})\right)$ or $\mathrm{s} \in \mathrm{E}(\mathrm{B}(\mathrm{G}))$. Therefore $\mathrm{E}\left(\mathrm{T}_{\mathrm{B}}(\mathrm{G})\right)$ $\subseteq \mathrm{E}\left(\mathrm{T}_{\mathrm{b}}(\mathrm{G})\right) \cup \mathrm{E}(\mathrm{B}(\mathrm{G}))$.

Since $T_{b}(G)$ and $B(G)$ are subgraphs of $T_{B}(G)$ we have $\mathrm{E}\left(\mathrm{T}_{\mathrm{b}}(\mathrm{G})\right) \cup \mathrm{E}(\mathrm{B}(\mathrm{G})) \subseteq \mathrm{E}\left(\mathrm{T}_{\mathrm{B}}(\mathrm{G})\right)$.

Hence $E\left(T_{B}(G)\right)=E\left(T_{b}(G)\right) \cup E(B(G))$. This shows that $T_{B}(G)=T_{b}(G) \cup B(G)$, the union of the graph $T_{b}(G) \& B(G)$. Since $T_{B}(G)$ and $B(G)$ have no edge in common, we conclude that $T_{B}(G)=T_{b}(G) \oplus B(G)$, the ring sum of the graphs $T_{b}(G)$ $\& \mathrm{~B}(\mathrm{G})$.

(ii). By Theorem 1.15, we have that $T_{b}(G)=G \oplus B_{v}(G)$.

Now $\mathrm{T}_{\mathrm{B}}(\mathrm{G})=\mathrm{T}_{\mathrm{b}}(\mathrm{G}) \oplus \mathrm{B}(\mathrm{G})$ (by $\left.(\mathrm{i})\right)=\mathrm{G} \oplus_{\mathrm{B}_{\mathrm{V}}(\mathrm{G})} \oplus_{\mathrm{B}(\mathrm{G})}$ (by the Theorem 1.15)

The proof is complete.

The following Corollary answers the question "How many edges are there in total-block graph".

2.9 Corollary: $\left|\mathrm{E}\left(\mathrm{T}_{\mathrm{B}}(\mathrm{G})\right)\right|=|\mathrm{E}(\mathrm{G})|+\left|\mathrm{V}\left(\mathrm{B}_{1}\right)\right|+\ldots+\left|\mathrm{V}\left(\mathrm{B}_{\mathrm{m}}\right)\right|+$ $|\mathrm{E}(\mathrm{B}(\mathrm{G}))|$, where $\mathrm{B}_{1}, \mathrm{~B}_{2}, \ldots, \mathrm{B}_{\mathrm{m}}$ are the blocks of $\mathrm{G}$.

Proof: By Theorem 2.8, we have that $\mathrm{T}_{\mathrm{B}}(\mathrm{G})=\mathrm{T}_{\mathrm{b}}(\mathrm{G}) \oplus$ $\mathrm{B}(\mathrm{G})$. Therefore $\left|\mathrm{E}\left(\mathrm{T}_{\mathrm{B}}(\mathrm{G})\right)=\right| \mathrm{E}\left(\mathrm{T}_{\mathrm{b}}(\mathrm{G})\right)|+| \mathrm{E}(\mathrm{B}(\mathrm{G}))|=| \mathrm{E}(\mathrm{G}) \mid+$ $\left|\mathrm{V}\left(\mathrm{B}_{1}\right)\right|+\ldots+\left|\mathrm{V}\left(\mathrm{B}_{\mathrm{m}}\right)\right|+|\mathrm{E}(\mathrm{B}(\mathrm{G}))|=$ (by the Theorem 1.9). The proof is complete.

A straightforward observation leads to the following.

2.10 Corollary: Let v be a vertex in a given graph G. Then (i) Degree of $v$ in $T_{B}(G)=$ degree of $v$ in $T_{b}(G)$, (ii) Degree of $v$ in $T_{B}(G)=\delta(v)+\mid\{B / B$ is a block in $G$ such that $v$ lies in $\mathrm{B}\} \mid$.

2.11 Corollary: Let $\mathrm{G}$ be a graph and v a vertex in $\mathrm{G}$.

(i) degree of $v$ in $T_{B}(G) \geq$ degree of $v$ in $G$

(ii) degree of $\mathrm{v}$ in $\mathrm{T}_{\mathrm{B}}(\mathrm{G})=$ degree of $\mathrm{v}$ in $\mathrm{G} \Leftrightarrow$ degree of $\mathrm{v}$ in $T_{b}(G)=$ degree of $v$ in $G \Leftrightarrow v$ is not contained in any block of $\mathrm{G}$.

\section{ACKNOWLEDGMENTS}

The first author acknowledges the UGC, New Delhi for the grant F. No. 34-136/2008 (SR) dated 30th Dec 2008. The third author acknowledges the Manipal University for the kind encouragement.

\section{REFERENCES}

[1] Arumugam S. and Ramachandran S. "Invitation to Graph Theory", Scitech Publications (India) Pvt. Ltd, Chennai, (2001).

[2] Bondy J. A. and Murty U. S. R. "Graph Theory with Applications", The Macmillan Press Ltd, (1976).

[3] Harary F. "Graph Theory", Addison-Wesley Publishing Company, USA (1972).

[4] Kulli V. R. "Minimally Non outer planar Graphs: A survey", (in the Book: "Recent Studies in Graph theory" ed: V. R. Kulli), Vishwa International Publication, (1989) 177-189.

[5] NarsingDeo "Graph Theory with Applications to Engineering and Computer Science", Prentice Hall of India Pvt. Ltd, New Delhi (1997).

[6] SatyanarayanaBh. and Syam Prasad K. "An Isomorphism Theorem on Directed Hypercubes of Dimension n", Indian J. Pure \& Appl. Math 34 (10) (2003) 1453-1457.

[7] SatyanarayanBh., and Syam Prasad K. "Discrete Mathematics and Graph Theory", Prentice Hall of India, New Delhi, 2009.ISSN:978-81-203-3842-5.

[8] SatyanarayanaBh., Syam Prasad K. and Nagaraju D. "Prime Graph of a Ring", Journal of Combinatorics, Information and System Sciences, Vol. 35, 1-12, (2010).

[9] SatyanarayanaBh.,Syam Prasad K., and Srinivas K.B. 'Graph of a Nearring with respect to an Ideal', Communications in Algebra (USA) Taylor \& Francis (2010), 38 (5), 1957-1967.

[10] Syam Prasad K. and SatyanarayanaBh. "Finite Dimension in N-Groups and Fuzzy Ideals of Gamma Nearrings", VDM Verlag, (Germany), 2011. ISBN: 9783-639-36838-3 